\title{
Review: Perspectives on Web Services-Applying SOAP, WSDL and UDDIto Real-World Projects
}

\section{Journal Article}

\section{Author(s):}

Alonso, Gustavo

Publication date:

2004

\section{Permanent link:}

https://doi.org/10.3929/ethz-b-000423062

\section{Rights / license:}

In Copyright - Non-Commercial Use Permitted

\section{Originally published in:}

The Computer Journal 47(4), https://doi.org/10.1093/comjnl/47.4.505 


\section{Book Reviews}

O. Zimmermann, M. TOMLinson And S. PEuser

Perspectives on Web Services-Applying SOAP, WSDL and UDDI to Real-World Projects. Springer Verlag (2003). ISBN 3-540-00914-0. £34.50/€48.10. 650 pp. Hardbound.

This book focuses on SOAP, WSDL and UDDI as the basic infrastructure for Web services. In terms of contents, the book can be divided into two parts. The first part (Chapters 1,2,3,6 and 7) introduces these three specifications and discusses their advantages, including issues like adoption of technology, positioning of new projects and potential pitfalls. These chapters provide a good overview of Web services and cover all of the important aspects of the technology, including introductory material that is often taken for granted (e.g. the use of XML and XML schema). The chapter that describes the specifications is particularly thorough and should appeal to a wide audience. It certainly is the strongest and more valuable contribution of the book as it discusses details that are seldom covered by other books. These details, nevertheless, will be most useful to developers as the book does not go beyond the use of Web services as infrastructure - a limitation that explains comments such as those on page 20 that Web services are a replacement for EDI. This comment is true at the infrastructure level but not at the level of business logic (as initiatives like ebXML demonstrate). The book does not cover any other Web service specification beyond SOAP, WSDL and UDDI, although a few others are mentioned in passing.

The other half of the book (Chapters 4, 5 and the Appendix) is intended for developers interested in following a step by step guide on the use of WebSphere for developing Web services. This part does not differ much in content or presentation from any 'how to' guide of a commercial product: sequences of screen shots, instructions on where to click, what menu to pull down, etc. Comprehensive as it is, this part will have only a limited audience: this material will only make sense to developers familiar with WebSphere and it is based almost exclusively on IBM tools. The few exceptions are to illustrate functionality not yet supported in WebSphere or the appendix covering the development of clients in .NET. Being also based on the state of the art at the time of writing, it is not clear how long this information will remain useful as the specifications and the tools supporting them are evolving very quickly.

Overall, this is a book covering the most basic infrastructure of Web services. It will be most useful to developers familiar with IBM products and who want to get a comprehensive overview on Web services, including a description on how to use WebSphere for developing basic Web services. The book is very well written and organized. It is also easy to read despite the wealth of details and comprehensive explanations. The book also includes comments that indicate which information is useful to read depending on the interests and expertise of the reader. This greatly helps in navigating the book, particularly for readers interested in implementation details but who do not intend to develop a Web service themselves.

Gustavo Alonso

Swiss Federal Institute of Technology (ETHZ), Switzerland

\section{J. CHOMICKI, R. VAN DER MEYDEN AND G. SAAKE (EDS)}

Logics for Emerging Applications of Databases. Springer Verlag (2004). ISBN 3-540-00705-9. £50/€64.99. 368 pp. Hardbound.

This book is a collection of papers from the Dagstuhl Seminar 00291, describing current research on the interface between logics and databases. It has nine independent chapters written by leading researchers in the field.

The book addresses three leading themes: (i) how new applications in databases lead to research issues within existing logics, (ii) how new logics are developed in response to the needs of database applications, and (iii) how logicbased approaches relate to other formal approaches.

Chapter 1 shows that there is a correspondence between XML documents and automata and logics. It also provides connections between XML query mechanisms and existing logics. Chapter 2 presents a (long) survey on the semantics and complexity issues arising from queries in inconsistent databases. It describes the several research avenues pursued by its authors and their collaborators over the last five years including query transformation, repairs specification using logic programming and annotated logics, and the computational complexity of consistent query answers.

Chapter 3 is about update policies for nonmonotonic knowledge bases. A generic framework for the specification of update policies is presented and the behaviour of an agent updating its knowledge base is represented in a rulebased language. An implementation of the framework in the IMPACT agent platform is also discussed in order to show the effectiveness of the approach.

Chapter 4 surveys the subject of intelligent agents and associated logical foundations of (Multi-)Agent Systems. Various modalities such as knowledge, beliefs, desires, intentions and their corresponding logical formulations for single and multi-agents are presented. This chapter is a very good overview of modal logics for agent systems.

Chapter 5 presents some logical approaches to model workflow systems. Temporal logic (CTL), event algebras and concurrent transaction logic (CTR) were surveyed, with special emphasis given to the latter where consistency checking of workflow specifications and property verification are allowed. Other logical approaches to workflow modelling such as the ACTA approach are also briefly described.

Starting from the observation that approaches to data expiration range from ad hoc policies to sophisticated data analysis techniques, Chapter 6 presents a formalization of 